\title{
Novel homozygous $P A N K 2$ mutation identified in a consanguineous Chinese pedigree with pantothenate kinase-associated neurodegeneration
}

\author{
YAN-FANG LI ${ }^{1 *}$, HONG-FU LI ${ }^{2 *}$, YAN-BIN ZHANG ${ }^{3}$ and JI-MIN WU ${ }^{2}$ \\ Departments of ${ }^{1}$ Pediatrics and ${ }^{2}$ Neurology, Second Affiliated Hospital, Zhejiang University School \\ of Medicine, Hangzhou, Zhejiang 310009; ${ }^{3}$ Department of Neurology and Institute of Neurology, \\ First Affiliated Hospital, Fujian Medical University, Fuzhou, Fujian 350004, P.R. China
}

Received April 4, 2016; Accepted June 27, 2016

DOI: $10.3892 /$ br.2016.715

\begin{abstract}
Pantothenate kinase-associated neurodegeneration (PKAN) is a rare autosomal recessive neurodegenerative disorder resulting from pantothenate kinase 2 (PANK2) gene mutations. It is clinically characterized by early onset of extrapyramidal symptoms, with or without pigmentary retinopathy, optic atrophy and acanthocytosis. The specific radiographic appearance of PKAN is the eye-of-the-tiger sign. However, there are few studies regarding PKAN patients of Chinese Han ancestry. In the present study, a Chinese 20-year-old female with an 8-year history of unsteady walking and involuntary movements is described. Brain magnetic resonance imaging revealed eye-of-the-tiger sign. Following sequencing of PANK2, a novel homozygous c.863C $>$ T (p.P288L) mutation was identified in the patient and heterozygous c.863C $>\mathrm{T}$ was identified in her consanguineous parents. The absence of this mutation in the 1000 Genomes database, The Exome Aggregation Consortium, and 200 controls demonstrated that this mutation was probably pathogenic for PKAN in this family. In addition, the PANK2 c.863C $>\mathrm{T}$ mutation was predicted to be deleterious by SIFT, disease causing by Mutation Taster and probably damaging by PolyPhen2.
\end{abstract}

\section{Introduction}

Pantothenate kinase-associated neurodegeneration (PKAN), also termed Hallervorden-Spatz syndrome (HSS), is a rare autosomal recessive neurodegenerative disorder resulting from mutations of the pantothenate kinase 2 (PANK2) gene (1). It

Correspondence to: Dr Ji-Min Wu, Department of Neurology, Second Affiliated Hospital, Zhejiang University School of Medicine, 88 Jiefang Road, Hangzhou, Zhejiang 310009, P.R. China

E-mail: jmwu@zju.edu.cn

*Contributed equally

Key words: pantothenate kinase-associated neurodegeneration, pantothenate kinase 2, homozygous mutation, eye-of-the-tiger sign is the most common form of neurodegeneration with brain iron accumulation (NBIA) (2). Clinically, it is characterized by childhood onset of dystonia, dysarthria, rigidity, and choreoathetosis, with or without pigmentary retinopathy, optic atrophy, and acanthocytosis (3). Approximately one-third of the PKAN patients showed cognitive decline or dementia (3). In typical PKAN, symptoms present within the first decade of life and usually rapidly progress, culminating in early mortality. However, in atypical PKAN, the onset of extrapyramidal symptoms is later, and the progression is slower and more variable. The characteristic imaging pattern of PKAN is eye-of-the-tiger sign, which consists of bilateral hyper-intensity within surrounding hypo-intensity in the globus pallidus in T2-weighted images (4).

The prevalence of PKAN is low, particularly in the Chinese population. To the best of our knowledge, no $>30$ PKAN cases of Chinese ancestry have been documented so far (5-10). The current study describes a PKAN patient of a consanguineous Chinese pedigree who exhibited the novel homozygous PANK2 mutation.

\section{Materials and methods}

Subjects. The current study was approved by the ethics committee of the Second Affiliated Hospital, Zhejiang University School of Medicine (Hangzhou, China). A single PKAN patient from a consanguineous Chinese pedigree was recruited. Written informed consent was obtained from the participants prior to blood sample collection. In addition, 200 healthy individuals of Chinese ancestry were recruited as control subjects. The neurological examinations (including consciousness, recognition, gait, cranial nerve, motor, sensory, reflex, pathological signs and coordination) and clinical evaluations (including age at onset, symptoms and signs, disease course and treatment) were performed. Brain magnetic resonance imaging (MRI) was conducted in the PKAN patient. Blood samples $(3 \mathrm{ml})$ were collected for laboratory inspection and gene sequencing.

Mutation analysis. After obtaining informed consent, genomic DNA was extracted from $3 \mathrm{ml}$ peripheral blood 
A

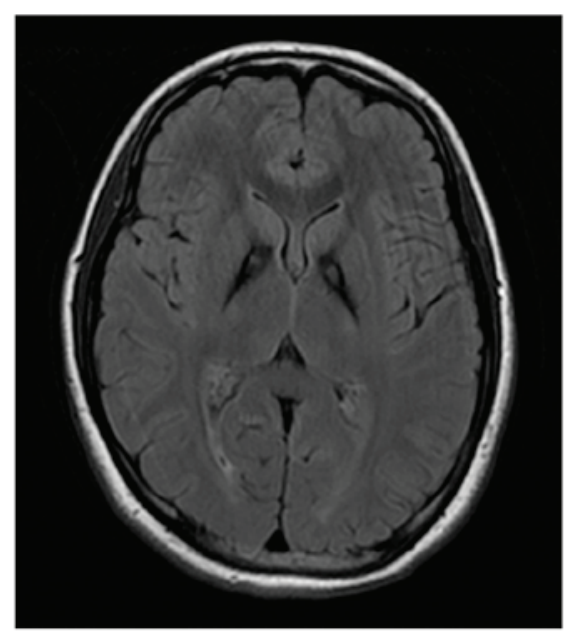

B

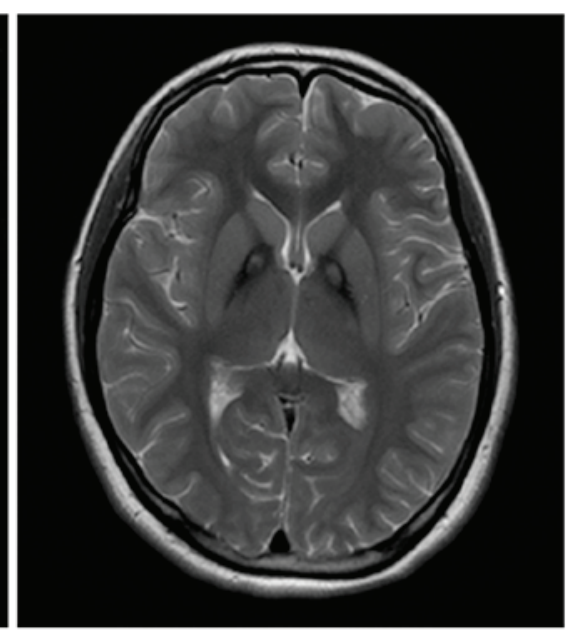

Figure 1. Axial magnetic resonance imaging demonstrates bilateral hyperintense pallidal areas on background areas of T2 shortening, termed eye-of-the-tiger sign. (A) T2-fluid attenuated inversion recovery and (B) T2-weighted.

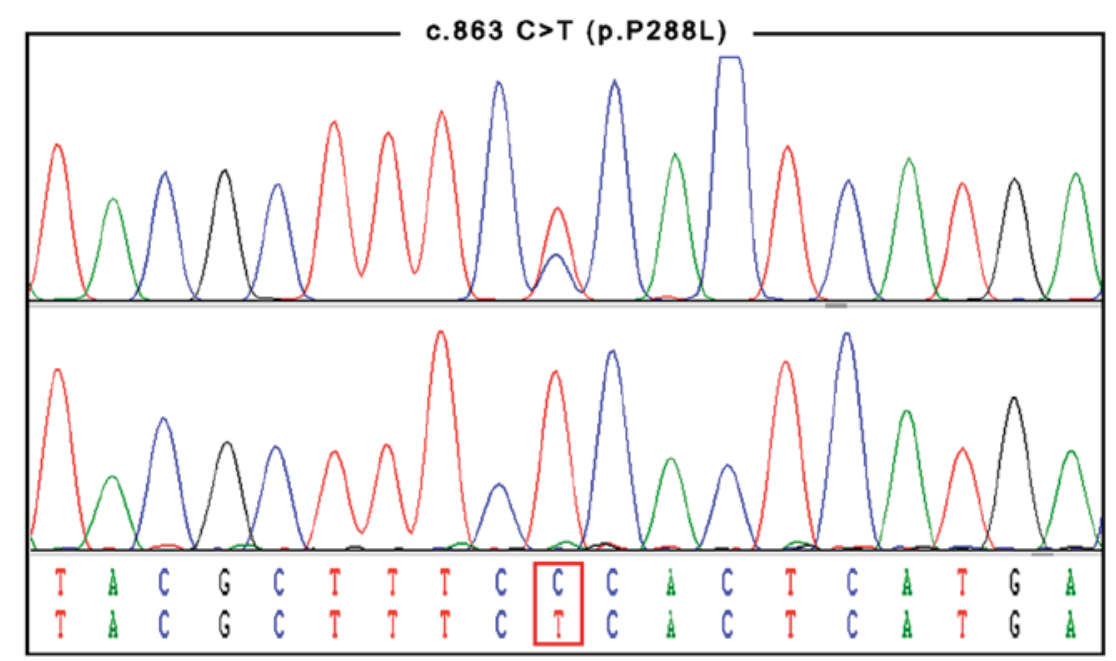

Figure 2. Chromatogram of the novel pantothenate kinase $2 \mathrm{c} .863 \mathrm{C}>\mathrm{T}$ mutation. Upper panel, the heterozygous sequence in the proband's consanguineous parents; lower panel, the homozygous mutation identified in the proband.

using a QIAamp DNA Blood Minikit (Qiagen GmbH, Hilden, Germany). Mutational investigation of PANK2 gene was performed by polymerase chain reaction (PCR) amplification and direct DNA sequencing, according to a previous study (1). Briefly, forward and reverse primers were designed to amplify each of the seven exons of PANK2 and all exons of PANK2 and the respective intron-exon boundaries were amplified by PCR according to a previous study (1). The purified PCR products were sequenced using an ABI 3730 Automated DNA Sequencer (Thermo Fisher Scientific, Inc., Waltham, MA, USA). The sequencing results were aligned to the NCBI human reference DNA sequence of PANK2 (Ensembl gene ID: ENSG00000125779; http://asia.ensembl.org/index. html). Amino acid and nucleotide changes were identified and numbered corresponding to their position within PANK2 mRNA. SIFT (http://sift.jcvi.org), PolyPhen2 (http://genetics. bwh.harvard.edu/pph2/) and Mutation Taster (http://www. mutationtaster.org) were used to predict the pathogenicity of the identified mutation.

\section{Results}

Clinical features of the patient. The proband was a 20-year-old female who had an 8-year history of gait difficulties and involuntary movements. Her delivery and postnatal period was unremarkable. The patient had normal acquisition of developmental milestones until the age of 12 years when unstable walk and involuntary movements began to develop. Her upper limbs suffered moderate chorea and ballismus, which became accentuated by anxiety and diminished when she was calm. Clonazepam at dose of $0.5 \mathrm{mg}$ was prescribed and the symptoms were significantly relieved. Two years later, difficulties with walking and the involuntary movements were aggravated and the patient began to exhibit dysarthria. The patient experienced did not completely alleviate the symptoms. Oral administration of benzhexol ( $2 \mathrm{mg}$, three times per day) was effective in the first two months. When the patient sought medical care in the Department of Neurology, Second Affiliated Hospital, Zhejiang University School of Medicine 
(Hangzhou, China) in December 2015, she presented dysarthria, involuntary movements and unsteady gait.

On cranial nerve examination, the extra-ocular movements of the patient were normal without nystagmus and visual field deficits. Choreiform movement was observed in the face. Pronunciation was slurred with mild palilalia, although her tongue and soft palate were at midline. The patient's muscle strength was normal, although muscle tension was decreased in the extremities and trunk. Chorea was obvious in the patient's upper limbs. No sensory abnormalities were observed and tendon reflexes were brisk, while the Babinski sign was bilaterally negative. Finger-nose and heel-knee-shin test results were normal. The patient's gait was unstable although the Romberg's test result was negative. The patient's recognition was not impaired, with a mini-mental state examination score of 29/30. Laboratory findings included normal serum ferritin, ceruloplasmin, albumin and lipoprotein levels. The blood smear was negative for acanthocytes. A brain MRI revealed iron accumulation in the bilateral globus pallidus and putamen (Fig. 1).

The patient's father and mother were consanguineous, healthy and did not exhibit gait difficulty or dysarthria.

Identification of the novel PANK2 mutation. After sequencing of the PANK2 gene, a homozygous c.863C $>$ T mutation was identified in the proband (Fig. 2). This mutation was located in exon 2 of $P A N K 2$, causing a proline to leucine substitution at position 288 (p.P288L) of the enzyme. Her consanguineous parents were heterozygous carriers of c.863C $>\mathrm{T}$. The homozygous c.863C $>\mathrm{T}$ mutation was absent in the 1000 Genomes database (http://www.1000genomes.org/), The Exome Aggregation Consortium (ExAc; http://exac.broadinstitute. org/) and all 200 control individuals. The homozygous c. $863 \mathrm{C}>\mathrm{T}$ mutation of PANK2 was predicted to be deleterious by SIFT, disease causing by Mutation Taster and probably damaging by PolyPhen2.

\section{Discussion}

PKAN is a neurodegenerative disorder associated with excessive iron deposition in the globus pallidus (11). It was first described by German neuropathologists Hallervorden and Spatz in 1922, therefore is also referred to as HSS (12); however, was subsequently renamed PKAN (13). In the present study, a Chinese 20-year-old female with an 8-year history of unsteady walking and involuntary movements is described. A brain MRI revealed typical eye-of-the-tiger sign. After sequencing the $P A N K 2$, a homozygous c.863C $>$ T (p.P288L) mutation was identified in her genetic DNA. Further genetic investigations revealed a heterozygous c.863C $>\mathrm{T}$ mutation in her consanguineous parents. To the best of our knowledge, this mutation has not previously been reported; therefore it is considered to be a novel mutation. The absence of this mutation in the 1000 Genome database, ExAC, and 200 control individuals demonstrated that this mutation was potentially pathogenic for PKAN in this particular family. In addition, it was predicted to be deleterious by SIFT and Mutation Taster.

The patient described in the current study experienced early onset and presented with an unsteady walk, choreic movements, and dysarthria as primary symptoms. The cognitive impairment was not obvious, although the patient had experienced the disease for 8 years. These observations were consistent with two existing reports of Chinese PKAN patients $(5,6)$. Notably, cognitive impairment was not prominent in Asian PKAN patients $(2,14,15)$. By contrast, the prevalence of pyramidal signs, mental impairment, and Parkinsonism was higher in Caucasian patients (16). This discrepancy is potentially due to the ethnic differences.

To date, $>100$ mutations within PANK2 have been reported in PKAN cases worldwide (http://www.hgmd.org). The majority of PANK2 mutations are missense mutations, while duplication, deletion, splice-site mutation and exon deletion have also been reported (16). The majority of previously reported Chinese PKAN patients were detected to be carrying compound heterozygous mutations. In the present study, the patient carried a homozygous PANK2 mutation. Due to the Chinese single-child policy that commenced in 1980, this patient did not have any siblings. Therefore, co-segregation could not be conducted in this family. However, the early onset of extrapyramidal symptoms, characteristic eye-of-the-tiger sign in MRI, and identification of the homozygous $P A N K 2$ mutation revealed that this was a case of PKAN.

The mechanism by which mutations in the PANK2 gene lead to iron accumulation in the brain remains to be elucidated. $P A N K 2$ is an essential regulatory enzyme in coenzyme A biosynthesis (13). In addition, the protein encoded by $P A N K 2$ is crucial in the metabolism of pantothenate. A deficiency of PANK2 is reported to result in the accumulation of cysteine-containing substrates, which may undergo rapid auto-oxidation in the presence of iron, leading to free radical generation and cell damage (1). A limitation of the current study was that functional experiments were not performed to verify the pathogenicity of the identified mutation. Thus, further investigations are required.

\section{Acknowledgements}

The authors would like to thank the participants for their assistance and willingness to take part in the study. Funding for the present study was granted by the National Natural Science Foundation of China (grant no. 81500973) and Natural Science Foundation of Zhejiang Province (grant no. LY16H090006) to Hong-Fu Li.

\section{References}

1. Zhou B, Westaway SK, Levinson B, Johnson MA, Gitschier J and Hayflick SJ: A novel pantothenate kinase gene (PANK2) is defective in Hallervorden-Spatz syndrome. Nat Genet 28: 345-349, 2001

2. Hogarth P: Neurodegeneration with brain iron accumulation: Diagnosis and management. J Mov Disord 8: 1-13, 2015.

3. Tonekaboni SH and Mollamohammadi M: Neurodegeneration with brain iron accumulation: An overview. Iran J Child Neurol 8: 1-8, 2014.

4. Hayflick SJ, Westaway SK, Levinson B, Zhou B, Johnson MA, Ching KH and Gitschier J: Genetic, clinical, and radiographic delineation of Hallervorden-Spatz syndrome. N Engl J Med 348: 33-40, 2003.

5. Song XW, Wang YL, Shi YW, Deng WY, Chen SQ, Lin H, Yi YH and Liao WP: Clinical manifestations and detection of pantothenate kinase 2 gene mutation in a patient with Hallervorden-Spatz syndrome. Zhonghua Yi Xue Za Zhi 89: 3320-3323, 2009 (In Chinese). 
6. Zhang Y, Tang B, Guo J, Long Z, Xia K, Pan Q, Hu Z, Wu D, Tang J, Chen T and Yan X: Studies on PANK2 gene mutations in Chinese patients with Hallervorden-Spatz syndrome. Zhonghua Yi Xue Yi Chuan Xue Za Zhi 22: 189-191, 2005 (In Chinese).

7. Fung GP and Chan KY: Cervical myelopathy in an adolescent with Hallervorden-Spatz disease. Pediatr Neurol 29: 337-340, 2003.

8. Wu YR, Chen CM, Chao CY, Lyu RK and Lee-Chen GJ: Pantothenate kinase-associated neurodegeneration in two Taiwanese siblings: Identification of a novel PANK2 gene mutation. Mov Disord 24: 940-941, 2009.

9. Zhang YH, Tang BS, Zhao AL, Xia K, Long ZG, Guo JF, Westaway SK and Hayflick SJ: Novel compound heterozygous mutations in the PANK2 gene in a Chinese patient with atypical pantothenate kinase-associated neurodegeneration. Mov Disord 20: 819-821, 2005.

10. Ma LY, Wang L, Yang YM, Lu Y, Cheng FB and Wan XH: Novel gene mutations and clinical features in patients with pantothenate kinase-associated neurodegeneration. Clin Genet 87: 93-95, 2015.

11. Thomas M, Hayflick SJ and Jankovic J: Clinical heterogeneity of neurodegeneration with brain iron accumulation (Hallervorden-Spatz syndrome) and pantothenate kinase-associated neurodegeneration. Mov Disord 19: 36-42, 2004.
12. Hallervorden J and Spatz H: Eigenartige Erkrankung im extrapyramidalen System mit besonderer Beteiligung des Globus pallidus und der Substantia nigra. Z Ges Neurol Psychiatr 79: 254-302, 1922.

13. Popławska-Domaszewicz K, Florczak-Wyspiańska J and Kozubski W: Update on neurodegeneration with brain iron accumulation. Neurol Neurochir Pol 48: 206-213, 2014.

14. Hartig MB, Hörtnagel K, Garavaglia B, Zorzi G, Kmiec T, Klopstock T, Rostasy K, Svetel M, Kostic VS, Schuelke M, et al: Genotypic and phenotypic spectrum of PANK2 mutations in patients with neurodegeneration with brain iron accumulation. Ann Neurol 59: 248-256, 2006.

15. Yamashita S, Maeda Y, Ohmori H, Uchida Y, Hirano T, Yonemura K, Uyama E and Uchino M: Pantothenate kinase-associated neurodegeneration initially presenting as postural tremor alone in a Japanese family with homozygous N245S substitutions in the pantothenate kinase gene. J Neurol Sci 225: 129-133, 2004.

16. Lee CH, Lu CS, Chuang WL, Yeh TH, Jung SM, Huang CL and Lai SC: Phenotypes and genotypes of patients with pantothenate kinase-associated neurodegeneration in Asian and Caucasian populations: 2 cases and literature review. ScientificWorldJournal 2013: 860539, 2013. 